\title{
Patterns of healthcare utilization in patients with generalized anxiety disorder in general practice in Germany
}

\author{
Ariel Berger, M.P.H.* \\ Ellen Dukes, Ph.D.** \\ Hans-Ulrich Wittchen, Ph.D. ${ }^{* * *}$ \\ Robert Morlock, Ph.D. ** \\ John Edelsberg, M.D., M.P.H.* \\ Gerry Oster, Ph.D.* \\ * Policy Analysis, Inc. (PAI), USA \\ ** Pfizer Inc., USA \\ *** Institute of Clinical Psychology and \\ Psychotherapy, Technische Universitaet \\ Dresden, Dresden, Germany 01187
}

USA

GERMANY

\begin{abstract}
Background and Objectives: To describe patterns of healthcare utilization among patients with generalized anxiety disorder (GAD) in general practitioner (GP) settings in Germany.

Methods: Using a large computerized database with information from GP practices across Germany, we identified all patients, aged $\geq 18$ years, with diagnoses of, or prescriptions for, GAD (ICD-10 diagnosis code F41.1) between October 1, 2003 and September 30, 2004 ("GAD patients"). We also constituted an age- and sex-matched comparison group, consisting of randomly selected patients without any GP encounters or prescriptions for anxiety or depression (a common comorbidity in GAD) during the same period. GAD patients were then compared to those in the matched comparison group over the one-year study period.

Results: The study sample consisted of $3340 \mathrm{GAD}$ patients and an equal number of matched comparators. Mean age was 53.2 years; $66.3 \%$ were women. Over the 12 -month study period, GAD patients were more likely than matched comparators to have encounters for various comorbidities, including sleep disorders (odds ratio $[\mathrm{OR}]=6.75[95 \% \mathrm{CI}=5.31$, 8.57]), substance abuse disorders (3.91 [2.89, 5.28]), and digestive system disorders $(2.62[2.36,2.91])($ all $\mathrm{p}<0.01)$. GAD patients averaged 5.6 more GP encounters $(10.5$ [SD $=8.8$ ] vs 4.9 [5.7] for comparison group) and 1.4 more specialist referrals (2.3 [2.9] vs 0.9 [1.7]) (both $\mathrm{p}<0.01$ ). Only $58.3 \%$ of GAD patients received some type of psychotropic medication (i.e., benzodiazepines, antidepressants, and/or sedatives/hypnotics).
\end{abstract}


Conclusions: Patients with GAD in GP practices in Germany have more clinically recognized comorbidities and higher levels of healthcare utilization than patients without anxiety or depression.

Received 29 May 2008

Revised 12 December 2008

Accepted 28 January 2009

\section{Introduction}

Generalized anxiety disorder (GAD) is a chronic condition characterized by persistent worry or anxiety that occurs more days than not over a period of at least 6 months ${ }^{1}$, and is difficult to diagnose because of differing clinical presentations and the common occurrence of comorbid somatic diseases and/or mental disorders. Lifetime prevalence of GAD has been estimated to be $4 \%-6 \%^{2}$; oneyear prevalence is about $2 \%{ }^{3,4}$. GAD occurs infrequently among persons aged $<35$ years, and is two to three times more common among women ${ }^{3}$. It is the most common anxiety disorder among patients presenting to primary care physicians ${ }^{5,6}$, and is probably overrepresented in primary care settings, with point prevalence estimates at least 2-3 times higher than that noted in the community 6,7 .

Most GAD patients experience other mental disorders during their lifetimes, most often major depressive disorder (MDD) ${ }^{8-10}$. One review estimated that over one year, $59 \%$ of patients with GAD had comorbid MDD, $71 \%$ had some form of depressive disorder, $29 \%$ had social phobia, $48 \%$ had a somatoform disorder, and $6 \%$ had alcohol abuse/dependence ${ }^{3}$. GAD patients also often have other comorbidities, including peptic ulcer disease, diabetes, and irritable bowel syndrome; cardiac and other somatic symptoms are also common ${ }^{11-19}$.
The impact of GAD on health-related quality of life and role functioning has been reported to be greater than that of $\mathrm{MDD}^{4}$. Also, because of comorbidities and frequent somatic complaints, GAD patients have been reported to have comparatively high levels of healthcare utilization and costs ${ }^{20,21}$. Detailed information on patterns of healthcare utilization among GAD patients is limited, however. One small survey-based study at community clinics in Canada reported that patients $(\mathrm{n}=219)$ who screened positive for GAD averaged 5.3 medical visits annually versus 3.4 visits for other patients ${ }^{22}$. GAD has been associated with a twofold increase in visits to primary care physicians as compared with patients without GAD, with or without depression, but with similar sociodemographic characteristics and chronic physical conditions $^{20,23}$. Among US patients with new episodes of anxiety (any type), mean total annual medical care costs have been reported to average $\$ 6,475$, or $\$ 2,138$ more than patients without anxiety ${ }^{24}$. One European study that used a health-economics model estimated that the mean total annual cost per case of GAD was $€ 1,804$; corresponding estimates were $€ 350$ for obsessive-compulsive disorder, €967 for panic disorder, $€ 937$ for social phobia, and $€ 3,826$ for depression $^{25}$.

In this study, we examine the characteristics of patients with GAD in GP settings in Germany -including their comorbidities, medica- 
tion use, and use of healthcare services- and compare them with those of age- and sexmatched patients without evidence of GAD also under GP care. This examination should improve knowledge of "real-world" patterns of healthcare utilization among patients with GAD under the care of GPs.

\section{Methods}

\section{Database}

Data were obtained from the IMS MediPlus - Disease Analyzer database, which provides patient-level information on diagnoses and treatments, and is comprised of about 4.2 million patient records and 75 million prescriptions over a 10 -year period. The database is compiled by sampling from approximately 900 GP practices throughout Germany and is designed to be representative of the general population in Germany. All patient identifiers in the database are fully encrypted.

Information in the database includes date of service, diagnoses (in ICD-10 format), actions taken (e.g., referrals to other providers [i.e., specialists], dispensing of sick notes [physician-excused absences from work]), and medications dispensed, including associated diagnoses. Selected demographic information is also available. All patient-level data can be arrayed chronologically to provide a detailed, longitudinal profile of all medical and pharmacy services rendered by participating GPs.

\section{Sample Selection}

The study sample consisted of all patients, aged $\geq 18$ years, with any GP visits resulting in a diagnosis of -or prescription for-GAD
(ICD-10 diagnosis code F41.1) between October 1, 2003 and September 30, 2004 ("study period") (ICD-10 diagnoses were used in lieu of criteria set forth in the Diagnostic and Statistical Manual of Mental Disorders, $4^{\text {th }}$ Edition [DSM-IV], as information on the latter was not available in the database). A comparison group also was constituted, consisting of randomly selected patients without any GP encounters or prescriptions for anxiety (e.g., panic disorder, obsessive-compulsive disorder, post-traumatic stress disorder, phobias, GAD) or depressive disorders (e.g., dysthymic disorder, adjustment disorder with depression, bipolar depression, major depressive disorder) during the same period, matched 1:1 to GAD patients based on age (within 5 years) and sex. Comparison group patients were selected from among all patients without evidence of anxiety or depression (rather than anxiety alone), as GAD patients often receive diagnoses of other anxiety or mood disorders (sometimes in lieu of a GAD diagnosis). All GP encounters were compiled for all GAD and comparison group patients over the study period.

\section{Measures}

We examined the prevalence of selected comorbidities over the 12-month study period, including: (1) neoplasms; (2) circulatory system disorders; (3) digestive system disorders; (4) somatoform disorders; (5) substance abuse disorders; and (6) sleep disorders. Patients were deemed to have these conditions if they had any encounters during the study period with the corresponding diagnosis code(s).

We examined the number of patients receiving selected medications during the study period, with particular focus on psychotropic medications (i.e., benzodiazepines, antide- 
pressants, sedatives/hypnotics); we also examined the use of healthcare services (i.e., GP visits, referrals to other healthcare providers, hospitalizations) and receipt of sick notes (physician-excused absences from work [diagnosis unspecified]). These measures were examined in terms of the number of patients receiving each service, and the total number of times it was rendered.

\section{Analyses}

Use of prescription medication was examined in terms of the numbers of patients who received various medications and the numbers of prescriptions thereof. As many of the measures of interest were not normally distributed (e.g., number of prescriptions received, number of visits), statistical significance of differences between GAD patients and matched comparators was assessed using Wilcoxon signed-rank tests for continuous measures, and McNemar's or Bowker's tests, as appropriate, for differences in categorical measures. All analyses were conducted using PC-SAS ${ }^{\circledR}$ v.8.4 $4^{26}$.

\section{Results}

The study sample consisted of 6680 patients-3340 with GAD, and an equal number of matched comparators. Mean $( \pm \mathrm{SD})$ age was 53.2 (17.7) years; $66.3 \%$ were women.

One-fifth of GAD patients had evidence of other anxiety disorders during the study period, and $28.8 \%$ had comorbid depression; $44.6 \%$ had comorbid anxiety and/or depression (Table I) (comparison group patients, by definition, could not have these disorders). GAD patients were more likely than matched comparators to have other mental disorders, including neurasthenia, sleep disorders, and substance abuse disorders (all $\mathrm{p}<0.01$ ); they also were more likely to have comorbidities, including digestive system disorders and circulatory system disorders (both $\mathrm{p}<0.01$ ).

During the study period, $58.3 \%$ of GAD patients received one or more psychotropic medications; $30.8 \%$ received benzodiazepines, and $37.2 \%$ received antidepressants. GAD patients were more likely than matched

Table I

Prevalence of concurrent somatic diseases and mental disorders among GAD patients and age- and sex-matched comparison group*

\begin{tabular}{lcccc} 
Comorbid Condition & $\begin{array}{c}\text { GAD Patients } \\
(\mathrm{N}=3,340)\end{array}$ & $\begin{array}{c}\text { Comparison } \\
\text { Group }(\mathrm{N}=3,340)\end{array}$ & OR (95\% CI) & $P$-Value \\
\hline Psychiatric disorders & & & & \\
Anxiety disorders & & & - & - \\
$\quad$ Panic disorder & $144(4.3)$ & - & - & - \\
OCD & $13(0.4)$ & - & - & - \\
PTSD & $7(0.2)$ & - & - & - \\
Phobias & $4(0.1)$ & - & - & - \\
$\quad$ Social phobia & $11(0.3)$ & - & - & - \\
$\quad$ Agoraphobia & $53(1.6)$ & - & - & - \\
All other phobias & $67(2.0)$ & - & - & - \\
Any phobia & $3,340(100.0)$ & - & - & - \\
GAD & $634(19.0)$ & - & - & - \\
Other anxiety disorder & $3,340(100.0)$ & - & & \\
Any anxiety disorder & & & & \\
\hline
\end{tabular}


Table I (continue)*

Comorbid Condition

GAD Patients Comparison OR $(95 \% \mathrm{CI}) \quad P$-Value $(\mathrm{N}=3,340) \quad$ Group $(\mathrm{N}=3,340)$

\begin{tabular}{|c|c|c|c|c|}
\hline \multicolumn{5}{|l|}{ Depression disorders } \\
\hline Dysthymic disorder & $44(1.3)$ & - & - & - \\
\hline Adjustment disorder with depression & $24(0.7)$ & - & - & - \\
\hline Bipolar depression & $1(0.0)$ & - & - & - \\
\hline MDD & $107(3.2)$ & - & - & - \\
\hline Unspecified depression & $856(25.6)$ & - & - & - \\
\hline Any depression & $962(28.8)$ & - & - & - \\
\hline Substance use disorders & $205(6.1)$ & $55(1.6)$ & $3.91(2.89,5.28)$ & $<0.01$ \\
\hline Sleep disorders & $485(14.5)$ & $82(2.5)$ & $6.75(5.31,8.57)$ & $<0.01$ \\
\hline Somatoform disorders & $547(16.4)$ & $101(3.0)$ & $6.28(5.05,7.81)$ & $<0.01$ \\
\hline Neurasthenia & $218(6.5)$ & $32(1.0)$ & $7.22(4.96,10.49)$ & $<0.01$ \\
\hline \multicolumn{5}{|l|}{ Number of } \\
\hline \multicolumn{5}{|l|}{ Concurrent anxiety disorders** } \\
\hline 0 & $2,532(75,8)$ & - & - & - \\
\hline 1 & $752(22,5)$ & - & - & - \\
\hline 2 & $54(1,6)$ & - & - & - \\
\hline$\geq 3$ & $2(0,1)$ & - & - & - \\
\hline \multicolumn{5}{|l|}{ Concurrent depression disorders } \\
\hline 0 & $2,378(71,2)$ & - & - & - \\
\hline 1 & $894(26,8)$ & - & - & - \\
\hline 2 & $66(2,0)$ & - & - & - \\
\hline & $2(0,1)$ & - & - & - \\
\hline \multicolumn{5}{|c|}{ Concurrent anxiety and/or depression disorders** } \\
\hline 0 & $1,852(55,4)$ & - & - & - \\
\hline 1 & $1,124(33,7)$ & - & - & - \\
\hline 2 & $323(9,7)$ & - & - & - \\
\hline$\geq 3$ & $41(1,2)$ & - & - & - \\
\hline \multicolumn{5}{|l|}{ Any of above } \\
\hline \multicolumn{5}{|l|}{ Other disorders } \\
\hline Circulatory system disorders & $1,990(59.6)$ & $1,339(40.1)$ & $2.20(2.00,2.43)$ & $<0.01$ \\
\hline Musculoskeletal system disorders & $1,844(55.2)$ & $1,295(38.8)$ & $1.95(1.77,2.15)$ & $<0.01$ \\
\hline Symptoms, signs, ill-defined conditions & $1,729(51.8)$ & $891(26.7)$ & $2.95(2.66,3.27)$ & $<0.01$ \\
\hline Digestive system disorders & $1,519(45.5)$ & $806(24.1)$ & $2.62(2.36,2.91)$ & $<0.01$ \\
\hline Respiratory system disorders & $1,460(43.7)$ & $1,005(30.1)$ & $1.80(1.63,2.00)$ & $<0.01$ \\
\hline Eyes, nose, and throat & $1,004(30.1)$ & $717(21.5)$ & $1.57(1.41,1.76)$ & $<0.01$ \\
\hline Painful neuropathic disorders & $822(24.6)$ & $402(12.0)$ & $2.39(2.09,2.72)$ & $<0.01$ \\
\hline Diabetes & $463(13.9)$ & $316(9.5)$ & $1.54(1.32,1.79)$ & $<0.01$ \\
\hline $\begin{array}{l}\text { Anemia and other blood/ antibody } \\
\text { disorders }\end{array}$ & 388 (11.6) & 155 (4.6) & $2.70(2.23,3.28)$ & $<0.01$ \\
\hline Neoplasms & $328(9.8)$ & $198(5.9)$ & $1.73(1.44,2.08)$ & $<0.01$ \\
\hline
\end{tabular}

*All values represent number $(\%)$ of patients with encounters resulting in any of the above-listed diagnoses over a 12-month period.

**Excluding GAD.

GAD: Generalized anxiety disorder.

comparators to have received other medications, including cardiovascular drugs and opioids (both short- and long-acting formulations) (both $\mathrm{p}<0.01$ ) (Table II). GAD pa[7.8] vs 3.5 [5.0] for the comparison group); excluding psychotropic medications, corresponding numbers were 9.5 (12.4) and 4.8 tients averaged 4.3 more prescriptions $(7.8$ (8.3) respectively (both $\mathrm{p}<0.01$ ) (Figure). 
Table II

Number of GAD patients and patients in age- and sex-matched comparison group receiving various medications*

\begin{tabular}{|c|c|c|c|c|}
\hline \multirow[b]{2}{*}{ Medication Type } & \multicolumn{4}{|c|}{ Total } \\
\hline & $\begin{array}{l}\text { GAD Patients } \\
(\mathrm{N}=3,340)\end{array}$ & $\begin{array}{c}\text { Comparison } \\
\text { Group }(\mathrm{N}=3,340)\end{array}$ & OR $(95 \% \mathrm{CI})$ & $P$-Value \\
\hline Cardiovascular system & $1,566(46.9)$ & $1,048(31.4)$ & $1.93(1.75,2.13)$ & $<0.01$ \\
\hline Alimentary tract and metabolism & $1,380(41.3)$ & $852(25.5)$ & $2.06(1.85,2.28)$ & $<0.01$ \\
\hline NSAIDs \& COX-2 inhibitors & $1,348(40.4)$ & $828(24.8)$ & $2.05(1.85,2.28)$ & $<0.01$ \\
\hline Antidepressants & $1,241(37.2)$ & $0(0.0)$ & --- & $<0.01$ \\
\hline Benzodiazepines & $1,028(30.8)$ & $54(1.6)$ & $27.06(20.47,35.76)$ & $<0.01$ \\
\hline Systemic general anti-infectives & $873(26.1)$ & $673(20.1)$ & $1.40(1.25,1.57)$ & $<0.01$ \\
\hline Systemic hormonal preparations & $645(19.3)$ & $423(12.7)$ & $1.65(1.44,1.89)$ & $<0.01$ \\
\hline Dermatologicals & $560(16.8)$ & $375(11.2)$ & $1.59(1.38,1.83)$ & $<0.01$ \\
\hline Blood and blood forming organs & $535(16.0)$ & $311(9.3)$ & $1.86(1.60,2.16)$ & $<0.01$ \\
\hline \multicolumn{5}{|l|}{ Genito-urinary system and sex } \\
\hline hormones & $385(11.5)$ & $222(6.6)$ & $1.83(1.54,2.17)$ & $<0.01$ \\
\hline Antipsychotics & $369(11.0)$ & $32(1.0)$ & $12.84(8.92,18.49)$ & $<0.01$ \\
\hline Sedatives \& hypnotics & $368(11.0)$ & $65(1.9)$ & $6.24(4.77,8.16)$ & $<0.01$ \\
\hline Opioids & $264(7.9)$ & $115(3.4)$ & $2.41(1.92,3.01)$ & $<0.01$ \\
\hline Corticosteroids & $129(3.9)$ & $74(2.2)$ & $1.77(1.33,2.37)$ & $<0.01$ \\
\hline Antiepileptics & $108(3.2)$ & $30(0.9)$ & $3.69(2.45,5.54)$ & $<0.01$ \\
\hline Muscle relaxants & $63(1.9)$ & $42(1.3)$ & $1.51(1.02,2.24)$ & 0.04 \\
\hline Anti-migraine medications & $65(1.9)$ & $35(1.0)$ & $1.87(1.24,2.83)$ & $<0.01$ \\
\hline \multicolumn{5}{|l|}{ Antineoplastic and } \\
\hline immunomodulating & $38(1.1)$ & $30(0.9)$ & $1.27(0.78,2.05)$ & 0.33 \\
\hline Any of above & 2,981 (89.3) & $2,295(68.7)$ & $3.78(3.31,4.31)$ & $<0.01$ \\
\hline None of above & $359(10.7)$ & $1,045(31.3)$ & $0.26(0.23,0.30)$ & $<0.01$ \\
\hline \multicolumn{5}{|c|}{ Number of psychotropic medications received** } \\
\hline 0 & $1,393(41.7)$ & $3,222(96.5)$ & $0.03(0.02,0.03)$ & --- \\
\hline 1 & $1,206(36.1)$ & $113(3.4)$ & $16.14(13.21,19.72)$ & --- \\
\hline 2 & $477(14.3)$ & $5(0.1)$ & $111.13(45.98,268.60)$ & --- \\
\hline$\geq 3$ & $264(7.9)$ & $0(0.0)$ & --- & --- \\
\hline Any of above & $1,947(58.3)$ & $118(3.5)$ & $38.16(31.37,46.44)$ & $<0.01$ \\
\hline
\end{tabular}

*All values represent number $(\%)$ of patients receiving the above-listed medications over a 12-month period. **Consists of antidepressants, benzodiazepines, sedatives \& hypnotics

GAD: Generalized anxiety disorder; NSAID: Nonsteroidal anti-inflammatory drug; COX: Cyclo-oxygenase

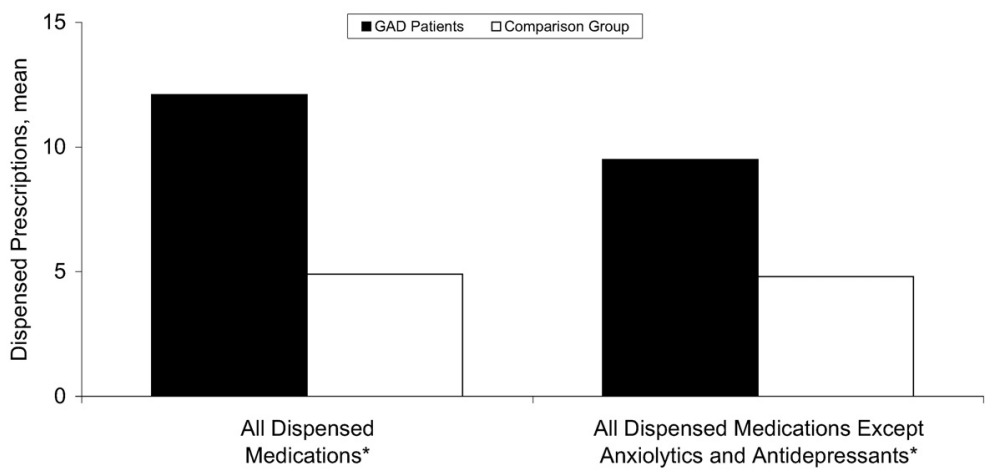

Figure. Mean number of dispensed prescriptions among GAD patients and patients in age- and sex-matched comparison group *Differences between GAD patients and comparison group were significant $(\mathrm{p}<0.01)$ 
Use of psychotropic medications by GAD patients increased with age: $51.7 \%$ among those aged $<45$ years, $57.8 \%$ among those aged $45-$ 64 years, and $66.7 \%$ among those aged $\geq 65$ years $(\mathrm{p}<0.01)$; there also was a statistically significant difference by gender $(60.6 \%$ among women vs $53.7 \%$ among men $[\mathrm{p}<0.01])$.
GAD patients averaged 5.6 additional GP visits, 1.4 more referrals, 0.1 additional hospitalizations, and 0.5 more sick notes (physician-excused absences from work) than matched comparators over 12 months (all p < 0.01) (Table III).

Table III

Numbers of provider visits, referrals, hospitalizations, and sick notes among GAD patients and patients in ageand sex-matched comparison group*

\begin{tabular}{|c|c|c|c|c|}
\hline & & $\begin{array}{l}\text { GAD Patients } \\
(\mathrm{N}=3,340)\end{array}$ & $\begin{array}{l}\text { Comparison Group } \\
(\mathrm{N}=3,340)\end{array}$ & $P$-Value \\
\hline GP visits & $\begin{array}{l}0 \\
1 \\
2 \\
3-4 \\
5-6 \\
7-9 \\
10-13 \\
>13 \\
\text { Mean (SD) } \\
\text { Median } \\
\text { Minimum } \\
\text { Maximum }\end{array}$ & 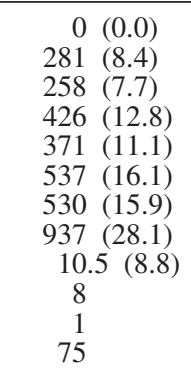 & $\begin{aligned} 0 & (0.0) \\
1,094 & (32.8) \\
482 & (14.4) \\
565 & (16.9) \\
362 & (10.8) \\
349 & (10.4) \\
237 & (7.1) \\
251 & (7.5) \\
4.9 & (5.7) \\
3 & \\
1 & \\
53 & \end{aligned}$ & $<0.01$ \\
\hline Referrals & $\begin{array}{l}0 \\
1 \\
2 \\
3 \\
\geq 4 \\
\text { Mean (SD) } \\
\text { Median } \\
\text { Minimum } \\
\text { Maximum }\end{array}$ & $\begin{array}{cc}1,096 & (32.8) \\
659 & (19.7) \\
437 & (13.1) \\
553 & (16.6) \\
595 & (17.8) \\
2.3(2.9) \\
1 \\
0 \\
39\end{array}$ & $\begin{aligned} & 2,018(60.4) \\
& 670(20.1) \\
& 271(8.1) \\
& 238(7.1) \\
& 143(4.3) \\
& 0.9(1.7) \\
& 0 \\
& 0 \\
& 20\end{aligned}$ & $<0.01$ \\
\hline Hospitalizations & $\begin{array}{l}0 \\
1 \\
2 \\
3-4 \\
\geq 5 \\
\text { Mean (SD) } \\
\text { Median } \\
\text { Minimum } \\
\text { Maximum }\end{array}$ & $\begin{aligned} 3,040 & (91.0) \\
222 & (6.6) \\
50 & (1.5) \\
24 & (0.7) \\
4 & (0.1) \\
0.1 & (0.5) \\
0 & \\
0 & \\
10 & \end{aligned}$ & $\begin{aligned} 3,340 & (100.0) \\
0 & (0.0) \\
0 & (0.0) \\
0 & (0.0) \\
0 & (0.0) \\
0.0 & (0.0) \\
0 & \\
0 & \\
0 & \end{aligned}$ & $<0.01$ \\
\hline Sick notes & $\begin{array}{l}0 \\
1 \\
2 \\
3-4 \\
\geq 5 \\
\text { Mean (SD) } \\
\text { Median } \\
\text { Minimum } \\
\text { Maximum }\end{array}$ & $\begin{aligned} & 2,614(78.3) \\
& 269(8.1) \\
& 143(4.3) \\
& 151(4.5) \\
& 163(4.9) \\
& 0.7(1.8) \\
& 0 \\
& 0 \\
& 0 \\
& 18\end{aligned}$ & $\begin{array}{cc}2,907 & (87.0) \\
263 & (7.9) \\
79 & (2.4) \\
61 & (1.8) \\
30 & (0.9) \\
0.2 & (0.9) \\
0 & \\
0 & \\
14 & \end{array}$ & $<0.01$ \\
\hline
\end{tabular}

*Unless otherwise indicated, all values represent number (\%) of patients with each healthcare service over a 12-month period.

GAD: Generalized anxiety disorder; GP: General practitioner. 


\section{Discussion}

We found that GAD patients in GP practices in Germany had higher levels of healthcare utilization than patients without anxiety or depression (matched on age and sex). They averaged twice as many GP visits than matched comparators, and 3.5 times as many physician-excused absences from work; they also had a higher prevalence of clinically recognized comorbidities. Results from our study are consistent with findings reported earlier from community surveys and primary care settings. One small $(n=219)$ survey-based study of Canadian patients who screened positive for GAD at community clinics averaged 5.3 medical visits annually versus 3.4 visits for other patients ${ }^{22}$. Two prior studies have reported a two-fold difference in visits to primary care physicians among GAD patients as compared with patients without GAD, irrespective of comorbid depression, but with similar sociodemographic characteristics and chronic physical conditions ${ }^{20,23}$.

Forty percent of GAD patients did not receive psychotropic drugs from their GPs; only one-third received antidepressants (considered first-line therapy for GAD). Use of psychotropic medications was more common among women, and it increased with age. While levels of use of antidepressants are similar to those reported in previous studies $^{6,7}$, the high levels of use of benzodiazepines and analgesics noteworthy. Onethird of patients in our study received benzodiazepines, and one in ten received other sedatives/hypnotics. In a 2000 survey of 558 primary care physicians in Germany, only $17 \%$ of patients with recognized GAD were reported to have received sedatives (presumably, mostly benzodiazepines $)^{6}$. Whether this difference is an artifact of study design -our study was a retrospective analysis of computerized GP encounter data- while
Wittchen's study was a cross-sectional survey of physicians -or represents a secular increase in the use of benzodiazepines and other sedatives is unknown. Use of NSAIDs (including COX-2 inhibitors) and opioids in our study appears to be quite high (40\% and $8 \%$, respectively); we know of no other study that has examined the use of these agents in patients with GAD.

The existence of an etiologic link between GAD and various comorbidities is an open issue at this time. On the one hand, it is possible that some of these conditions may be causally related to GAD. Stressful life events (e.g., death of a loved one, divorce, diagnosis of a serious medical condition) are known to be precipitating factors for GAD. It has been argued that increasing rates of GAD among persons aged $>40$ years may be suggestive of a common etiologic link between GAD and various chronic comorbidities. A diagnosis of GAD also may reflect typical patterns of worry about declining health associated with advancing age. However, it is also important to note that our findings may simply reflect opportunistic case-finding. Patients with GAD frequently have somatic complaints, and workups for such symptoms may increase the likelihood of clinical recognition of underlying (but unrelated) diseases. Caution should therefore be exercised in the interpretation of our findings.

Our database contained information on more than 1.2 million patients, 3340 of whom were found to have evidence of GAD during the study period. The estimated annual prevalence of GAD in our study (i.e., $0.3 \%$ ) is markedly lower than the $2 \%$ reported in other studies ${ }^{3,4}$. The primary reason for this probably relates to the nature of the database, which only includes information recorded by GPs following patient encounters; patients who do not receive a GAD diagnosis from their GPs (irrespective 
of whether or not they are known by their GPs to have GAD), or whose GAD is treated exclusively by specialists, would not be reflected in our count. We note, though, that the demographic characteristics of GAD patients in our study are similar to those reported in other studies (i.e., two-thirds women, three-quarters aged $\geq 40$ years).

Almost thirty percent of GAD patients in our study also had been diagnosed with depression by their GPs, which is within the range (16-39\%) reported in previous re$\operatorname{search}^{9,20}$. The prevalence of digestive disorders, somatoform disorders, sleep disorders, and symptoms, signs, and ill-defined conditions also was high among GAD patients, consistent with earlier findings suggesting that GAD is commonly associated with various somatic disorders, including chest pain, irritable bowel syndrome, and heart disease $\mathrm{e}^{11,14,15,27-30}$.

Evidence-based guidelines recommend that selective serotonin receptor inhibitors (SSRIs), tricyclic antidepressants (TCAs), benzodiazepines, and venlafaxine be used for the treatment for GAD ${ }^{31}$. Many GAD patients in our study, however, had no record of receipt of such medications from their GPs. Patients in our study may have been prescribed such medications by specialists, however (the database only contains information on GP prescribing).

Some important limitations of our study should be noted. For one, information in the study database is limited to GP encounters. Given the high prevalence of comorbidities, it is reasonable to expect that GAD patients also had encounters with specialists. Furthermore, records of referrals, sick notes, and hospitalizations available in the study database are complete only to the extent that GPs recorded this information.
Study subjects were selected based on GP-recognized GAD, as evidenced by an encounter with an ICD-10 diagnosis code for this condition during the period of interest. Because we did not have access to patients' medical records, we cannot ascertain whether GPs used established DSM-IV criteria in rendering a diagnosis of GAD. Moreover, our database was limited to information on GP encounters. Thus, patients whose GAD was under the exclusive care of a specialist would not have been included in our GAD population -and in fact may have been selected for the comparison group if they saw their GP for other health problems. The degree to which any resulting misclassification in either or both patient groups impacted our findings is unknown.

In conclusion, our findings suggest that patients with GAD who are receiving care in GP settings in Germany have more clinically recognized comorbidities and higher levels of healthcare utilization than (ageand sex-matched) patients without evidence of anxiety or depression. Effective treatments for GAD may lead to reductions in healthcare utilization to the extent that these high levels may be attributable to this disorder. Further research is needed to better quantify the relationship between GAD and excess levels of healthcare utilization.

\section{Acknowledgements}

All authors (Ariel Berger, M.P.H., Ellen Dukes, Ph.D., Hans-Ulrich Wittchen, Ph.D., Robert Morlock, Ph.D., John Edelsberg, M.D., M.P.H., Gerry Oster, Ph.D.) helped with all aspects of this study (i.e., conceptualization and design of study, analysis and interpretation of data, manuscript preparation and review). 
Funding for this research was provided by Pfizer Inc., New York, NY. Dr. Dukes and Dr. Morlock, employees of Pfizer Inc. at the time this research was undertaken as well as co-authors of this manuscript, were involved with the design of the study, data analysis and interpretation, manuscript preparation, and publication decisions. Mr. Berger, Dr. Edelsberg, and Dr. Oster are employees of Policy Analysis, Inc., who were paid consultants to Pfizer in consultation with the development of this manuscript.

\section{References}

1. American Psychiatric Association. (2000) Diagnostic and Statistical Manual of Mental Disorders, Fourth Edition, Text Revision. Washington DC: American Psychiatric Association; 1992.

2. Kessler R, Berglund P, Demler O, Jin R, Merikangas K, Walters E. Lifetime prevalence and age of onset distributions of DSM-IV disorders in the National Comorbidity Survey Replication. Arch Gen Psychiatry 2005; 62(6): 593-602.

3. Lieb R, Becker E, Altamura C. The epidemiology of generalized anxiety disorder in Europe. Eur Neuropsychopharmacol European Neuropsychopharmacology 2005; 15(4): 445-452.

4. Wittchen HU, Carter RM, Pfister H, Montgomery SA, Kessler RC. Disabilities and quality of life in pure and comorbid generalized anxiety disorder and major depression in a national survey. Int Clin Psychopharmacol 2000; 15(6): 319-328.

5. Ormel J, VonKorff M, Ustun T, Pini S, Korton A, Oldehinkel T. Common mental disorders and disability across cultures: Results from the WHO collaborative study on psychological problems in general health care. JAMA 1994; 272(22): 1741-1748.

6. Wittchen HU, Kessler RC, Beesdo K, Krause P, Hofler M, Hoyer J. Generalized anxiety disorder and depression in primary care: Prevalence, recognition, and management. J Clin Psychiatry 2002; 63(Suppl 8): 24-34.

7. Monk-Jorgensen P, Allgulander C, Dahl AA, Foldager L, Holm M, Rasmussen IbIB, et al. Prevalence of generalized anxiety disorder in general practice in Denmark, Finland, Norway, and Sweden. Psych Services 2006; 57(12): 1738-1744.
8. Massion A, Warshaw M, Keller M. Quality of life and psychiatric morbidity in panic disorder and generalized anxiety disorder. Am J Psychiatry 1993; 150(4): 600-607.

9. Stein DJ. Comorbidity in generalized anxiety disorder: Impact and implications. J Clin Psychiatry 2001; 62(Suppl 11): S29-S34.

10. Wittchen HU, Zhao S, Kessler R, Eaton W. DSMIII-R generalized anxiety disorder in the National Comorbidity Survey. Arch Gen Psychiatry 1994; 51(5): 355-364.

11. Carter CS, Maddock RJ. Chest pain in generalized anxiety disorder. Int J Psychiatry Med 1991; 22(3): 291-298.

12. Goodwin R, Stein M. Generalized anxiety disorder and peptic ulcer disease among adults in the United States. Psychosom Med 2002; 64(6): 862-866.

13. Grigsby A, Anderson R, Freedland K, Clouse R, Lustman P. Prevalence of anxiety in adults with diabetes: A systematic review. J Psychosom Res 2002; 53(6): 1053-1060.

14. Kane FJ Jr., Harper RG, Wittels E. Angina as a symptom of psychiatric illness. South Med J 1988; 81(11): 1412-1416.

15. Logue MB, Thomas AM, Barbee JG, Hoehn-Saric R, Maddock RJ, Schwab J, et al. Generalized anxiety disorder patients seek evaluation for cardiological symptoms at the same frequency as patients with panic disorder. J Psychiatr Res 1993; 27(1): 55-59.

16. Lydiard R. An overview of generalized anxiety disorder: Disease state-appropriate therapy. Clin Ther 2000; 22(Suppl A): S3-S24.

17. Lydiard R, Fossey M, Marsh W, Ballenger J. Prevalence of psychiatric disorders in patients with irritable bowel syndrome. Psychosomatics 1993(3); 34(3): 229-234.

18. Pearce M, Mayou R, Klimes I. The management of atypical non-cardiac chest pain. Q J Med 1990; 76(3): 991-996.

19. Roy-Byrne P, Wagner A. Primary care perspectives on general anxiety disorder. J Clin Psychiatry 2004; 65(Suppl 13): S20-S26.

20. Wittchen HU. Generalized anxiety disorder: Prevalence, burden, and cost to society. Depress Anxiety 2002; 16(4): 162-171.

21. Hoffman DL, Dukes EM, Wittchen HU. Human and economic burden of generalized anxiety disorder. Depress Anxiety 2006; 1(1): 1-19.

22. Belanger L, Ladouceur R, Morin CM. Generalized anxiety disorder and health care use. Can Fam Physician 2005; 51(10): 1362-1363.

23. Kennedy BL, Schwab JL. Utilization of medical specialists by anxiety disorder patients. Psychosomatics 1997; 38(2): 109-112. 
24. Marciniak MD, Lage MJ, Dunayevich E, Russell JM, Bowman L, Landbloom RP, et al. The cost of treating anxiety: The medical and demographic correlates that impact total medical costs. Depress Anxiety Depression and Anxiety 2005; 21(4): 178-184.

25. Andlin-Sobocki P, Jonsson B, Wittchen HU, Olesen J. Cost of disorders of the brain in Europe. Eur J Neurol European Journal of Neurology 2005; 12(Supp 1): S1-S27.

26. SAS ${ }^{\circ}$ Proprietary Software, Release 8.4, SAS Institute Inc., Cary, NC.

27. Wulsin LR, Arnold LM, Hillard JR. Axis I disorders in ER patients with atypical chest pain. Int $\mathbf{J}$ Psychiatry Med 1991; 21(1): 37-46.

28. de Ruiter C, Garsson B, Rijken H, Kraaimaat F. The hyperventilation syndrome in panic disorder, agoraphobia and generalized anxiety disorder. Behav Res Ther 1989; 27(4): 447-452.

29. Maier W, Falkai P. The epidemiology of comorbidity between depression, anxiety disorders and somatic diseases. Int Clin Psychopharmacol 1999; 14(Suppl 2): S1-S6.

30. Sherbourne CD, Jackson CA, Meredith LS, Camp P, Wells KB. Prevalence of comorbid anxiety disorders in primary care outpatients. Arch Fam Med 1996; 5(1): 27-34.

31. Baldwin DS, Anderson IM, Nutt DJ, Bandelow B, Bond A, Davidson JRT, et al. Evidence-based guidelines for the pharmacological treatment of anxiety disorders: Recommendations from the British Association of Psychopharmacology. J Psychopharm 2005; 19(6): 567-596.

Address for correspondence:

Gerry Oster, Ph.D.

Policy Analysis, Inc. (PAI)

Four Davis Court

Brookline, Massachusetts 02445

USA

Tel.: 617-232-4400

Fax: 617-232-1155

E-mail: goster@pai2.com 